\title{
A Distributed Cortical Representation Underlies Crossmodal Object Recognition in Rats
}

\author{
Boyer D. Winters and James M. Reid \\ Department of Psychology, University of Guelph, Guelph, Ontario N1G 2W1, Canada
}

The mechanisms by which the brain integrates the unimodal sensory features of an object into a comprehensive multimodal object representation are poorly understood. We have recently developed a procedure for assessing crossmodal object recognition (CMOR) and object feature binding in rats using a modification of the spontaneous object recognition (SOR) paradigm. Here we show for the first time that rats are capable of spontaneous crossmodal object recognition when they are asked to recognize a visually presented object having previously only explored the tactile features of that object. Moreover, rats with bilateral perirhinal cortex $(\mathrm{PRh})$ lesions were impaired on the CMOR task and a visual-only, but not a tactile-only, version of SOR. Conversely, rats with bilateral posterior parietal cortex (PPC) lesions were impaired on the CMOR and tactile-only tasks but not the visual-only task. Finally, crossmodal object recognition ability was severely and selectively impaired in rats with unilateral lesions made to PRh and PPC in opposite hemispheres. Thus, spontaneous tactile-to-visual crossmodal object recognition in rats relies on an object representation that requires functional interaction between PRh and PPC, which appear to mediate the visual and tactile information-processing demands of the task, respectively. These results imply that, at least under certain conditions, the separate sensory features of an object are represented in a distributed manner in the cortex. The novel paradigm introduced here should be a valuable tool for further study of the neurobiological bases of crossmodal cognition and object feature binding.

\section{Introduction}

Human sensory experience is the product of complex stimulus representations resulting from the integration of information from various sensory modalities. The amalgamation of information from multiple sensory sources underlies object identification and localization, abilities that are essential in determining appropriate behavioral responses to objects and events (Stein and Meredith, 1993; Murray et al., 1998). For example, the ability to recognize a potential predator on the basis of auditory, olfactory, or visual cues confers great adaptive value. Moreover, humans are not alone in their ability to integrate information from multiple sensory modalities to aid object identification. Previous work has demonstrated robust crossmodal object recognition (CMOR) in apes and monkeys (Davenport and Rogers, 1970; Cowey and Weiskrantz, 1975; Elliott, 1977; Jarvis and Ettlinger, 1977; Murray and Mishkin, 1985).

An important unresolved question concerns how the brain integrates unimodal object information into a coherent multisensory object representation. Specifically, there has been much debate over whether this function is accomplished by comparison between separate modality-specific brain regions or the formation and storage of a unified multisensory representation in a polymodal brain area (Ettlinger and Wilson, 1990; Amedi et al.,

Received Dec. 7, 2009; revised Feb. 25, 2010; accepted March 26, 2010.

This research was supported by a National Sciences and Engineering Research Council of Canada Discovery Grant to B.D.W. We thank Emily Marshall for behavioral scoring.

Correspondence should be addressed to Dr. Boyer D. Winters, Department of Psychology, University of Guelph, Guelph, 0N N1G 2W1, Canada. E-mail: bwinters@uoguelph.ca.

D0I:10.1523/JNEUROSCI.6073-09.2010

Copyright $\odot 2010$ the authors $\quad 0270-6474 / 10 / 306253-09 \$ 15.00 / 0$
2005; Lacey et al., 2007; Stein and Stanford, 2008). Although electrophysiological studies indicate multisensory neurons throughout the cortex (Stein and Stanford, 2008), robust demonstration of a singular brain region whose damage selectively impairs crossmodal object recognition has yet to be made. Indeed, studies with brain-damaged monkeys have yielded equivocal results in this regard (Sahgal et al., 1975; Ettlinger and Wilson, 1990; Murray et al., 1998; Goulet and Murray, 2001).

Clearly, further research is required to uncover the neural bases of crossmodal object recognition. Although the abilities of rats to learn configural associations between discrete stimuli from different sensory modalities have previously been studied (Over and Mackintosh, 1969; Tomie and Whishaw, 1990; Lipton et al., 1999; Sakata et al., 2004; Botly and De Rosa, 2007, 2009a,b), no paradigm, to our knowledge, has demonstrated the capacity of rats to recognize a unitary object in a task that requires integration of multisensory information about that object (Jeffery, 2007). Demonstration of this ability in rats would provide a valuable model for the study of the neurobiological underpinnings of crossmodal cognition.

In the present study, we assessed the roles of the perirhinal (PRh) and posterior parietal (PPC) cortices in crossmodal object recognition in rats. Previous research indicates that $\mathrm{PRh}$ is particularly important for object information processing (Buckley and Gaffan, 2006; Murray et al., 2007; Winters et al., 2008). Indeed, findings from humans and monkeys suggest that PRh is involved in crossmodal analysis of object features (Goulet and Murray, 2001; Taylor et al., 2006; Holdstock et al., 2009). The rat PPC receives projections from somatosensory, auditory, and visual areas (Reep et al., 1994) and may therefore be important for 
processing multimodal sensory information (Zhou and Fuster, 2000). Here we show for the first time that rats are capable of tactile-to-visual crossmodal object recognition and that different cortical regions underlie different aspects of the object representation required for this cognitive function.

\section{Materials and Methods}

Subjects. The subjects were 119 adult male Long-Evans rats (Charles River) weighing $\sim 300-350 \mathrm{~g}$ at the onset of each experiment. Rats were housed in pairs and maintained on a reverse light/dark cycle (7:00 A.M. lights off; 7:00 P.M. lights on). All behavioral testing occurred during the dark phase of the cycle, and lighting conditions in the testing room were specific to the task being run (see below). During each experiment, rats received $20 \mathrm{~g}$ of rodent chow each evening to maintain $85-90 \%$ of freefeeding body weight. Water was available ad libitum throughout the experiments. All procedures adhered to the guidelines of the Canadian Council on Animal Care and were approved by the Animal Care Committee at the University of Guelph.

Surgical procedures. Before and during all surgeries, rats were deeply anesthetized with isofluorane (Benson Medical Industries) inhalation anesthetic. Animals also received a systemic subcutaneous injection of the analgesic meloxicam $(5 \mathrm{mg} / \mathrm{ml}$; Boehringer Ingelheim) before surgery. They were then placed in a stereotaxic frame (Kopf Instruments) with the incisor bar set to $-3.3 \mathrm{~mm}$ for posterior parietal cortex (PPC) lesions and $+5.0 \mathrm{~mm}$ for perirhinal cortex (PRh) lesions. The scalp was cut and retracted to expose the skull, and craniotomies were performed directly above the target region.

Rats in the bilateral PRh lesion experiment were randomly assigned to receive either bilateral excitotoxic PRh lesions $(n=7)$ or sham surgery $(n=9)$. For the PRh lesions, infusions of $0.2 \mu \mathrm{l}$ of $0.9 \mathrm{M}$ NMDA (Sigma) dissolved in phosphate buffer, $\mathrm{pH}$ 7.4, were made through a $1 \mu \mathrm{l} \mathrm{Ham}$ ilton syringe into three sites in each hemisphere. Each injection was delivered over a 2 min period, and the needle was left in situ for an additional $4 \mathrm{~min}$ before being withdrawn. The stereotaxic coordinates relative to ear-bar zero were as follows: anteroposterior $(\mathrm{AP})+3.9$, lateral $(\mathrm{L}) \pm 5.9$, dorsoventral (DV) +2.0 ; $\mathrm{AP}+2.4, \mathrm{~L} \pm 6.1, \mathrm{DV}+1.6$; and $\mathrm{AP}+0.6, \mathrm{~L} \pm 6.2, \mathrm{DV}+2.5$. Sham-operated rats underwent identical operations, except no solution was infused into the brain at the sites of needle placement.

Rats in the bilateral PPC lesion experiment were randomly assigned to receive either bilateral excitotoxic PPC lesions $(n=7)$ or sham surgery $(n=9)$. Four holes were drilled in the skull at the following locations relative to bregma: $\mathrm{AP}-4.0$ and $-4.7 ; \mathrm{L} \pm 2.5$ and \pm 3.7 (Fox et al., 2003). A single infusion was made at each of the four sites, using $0.9 \mathrm{M} \mathrm{NMDA}$ dissolved in phosphate buffer, $\mathrm{pH} 7.4$, administered $1.5 \mathrm{~mm}$ below the skull at the medial sites and $1.7 \mathrm{~mm}$ below the skull at the lateral sites. Each $0.2 \mu \mathrm{l}$ infusion was done over a period of $2 \mathrm{~min}$ and the needle left in place for an additional $2 \mathrm{~min}$. Sham-operated rats underwent identical operations, except no solution was infused into the brain at the sites of needle placement.

Rats in the crossed unilateral lesion experiment were randomly assigned to receive either crossed unilateral excitotoxic lesions of PRh and PPC $(n=9)$ or sham surgery $(n=11)$. The PRh and PPC lesions were conducted the same as described for the bilateral lesion experiments, except that rats received NMDA infusions into only one cortical region per hemisphere. Five rats received unilateral PPC lesions in the left hemisphere and unilateral PRh lesions in the right hemisphere; the sides were reversed for the remaining four rats. Sham-operated rats underwent identical operations, except no solution was infused into the brain at the sites of needle placement.

Rats in the separate unilateral lesion experiment were randomly assigned to receive unilateral excitotoxic lesions of PRh $(n=6,3$ left and 3 right) or PPC ( $n=6,3$ left and 3 right $)$ or sham surgery $(n=8 ; 4 \mathrm{PRh}$ shams and 4 PPC shams).

Finally, rats in the ipsilateral unilateral lesion experiment were randomly assigned to receive combined unilateral lesions of PRh and PPC in the same hemisphere ( $n=8 ; 4$ left and 4 right) or sham surgery $(n=10$; 5 left and 5 right).
At the completion of each surgery, the scalp was sutured, and animals recovered on heat pads for $1-2 \mathrm{~h}$ before being returned to their home cages. Rats were allowed to recover for $7-10 \mathrm{~d}$ before behavioral testing began.

Histology. After behavioral testing, rats were anesthetized by intraperitoneal injection of $2 \mathrm{ml}$ of Euthansol $(340 \mathrm{mg} / \mathrm{ml}$; Schering) and perfused transcardially with $100 \mathrm{ml}$ of PBS, pH 7.4, followed by $250 \mathrm{ml}$ of $4 \%$ neutral buffered formalin, pH 7.4 (EMD). The brains were removed, postfixed in $4 \%$ formalin at $4^{\circ} \mathrm{C}$ for $24 \mathrm{~h}$, and then immersed in $20 \%$ sucrose in PBS until they sank. Coronal sections $(60 \mu \mathrm{m})$ were cut using a cryostat through the extent of the lesioned area, and every fifth section was mounted on a gelatin-coated glass slide and stained with cresyl violet. Slides were examined under a light microscope to determine the extent of excitotoxin-induced damage. The extent of tissue damage in each targeted structure was quantified for each experiment by measuring the pixel volume of the drawings of the intact areas (PRh and PPC) from Paxinos and Watson (1998) and comparing these with the pixel volume of the drawings of the lesioned area for each rat. This was accomplished using ImageJ software (http://rsbweb.nih.gov/ij/). The percentage of lesioned area for each rat and the mean percentage lesioned area for each lesion group were calculated from these values. The boundaries for measuring PRh were determined from those previously defined by Burwell and colleagues (Burwell et al., 1995; Burwell and Amaral, 1998; Burwell, 2001). Although there is still some controversy regarding the exact boundaries of rat PPC, we referred to the region as defined in previous studies by thalamic and cortical connectivity (Chandler et al., 1992; Reep et al., 1994). Accordingly, we considered PPC to occupy the area of cortex located $\sim 3.5-5.0 \mathrm{~mm}$ caudal to bregma and $1.5-5.0 \mathrm{~mm}$ lateral to the midline suture (Bucci, 2009; Kesner, 2009; Reep and Corwin, 2009).

Spontaneous object recognition. Spontaneous object recognition (SOR) was conducted in a Y-shaped apparatus as described previously (Winters et al., 2004; Forwood et al., 2005). The Y-shaped apparatus had high homogenous white walls constructed from Plexiglas to prevent the rat from seeing out into the surrounding testing room. The apparatus walls were $40 \mathrm{~cm}$ high, and each arm was $27 \mathrm{~cm}$ long and $10 \mathrm{~cm}$ wide. The start arm contained a guillotine door $18 \mathrm{~cm}$ from the rear of the arm. This provided a start box area within which the rat could be confined at the start of a given trial. A video camera was mounted on a tripod above the apparatus to record all trials. Duplicate copies of "junk" objects made from plastic, ceramic, glass, and aluminum were obtained. The height of the objects ranged from 10 to $20 \mathrm{~cm}$, and they varied with respect to their visual and tactile qualities. All objects were affixed to the floor of the apparatus with an odorless reusable adhesive putty to prevent them from being displaced during testing. As far as could be determined, the objects had no natural significance for the rats. Before being placed in the apparatus, objects were always wiped with $50 \%$ ethanol.

Each experiment followed the same general procedure. Specific experimental manipulations are outlined below. For all lesion experiments, the experimenter was blind to the group assignments of subjects during all phases of behavioral testing and scoring. For white light (visual-only) conditions, the room was lit with a set of four overhead florescent lights, and a transparent plastic barrier was inserted $9 \mathrm{~cm}$ from the end of each exploration arm to prevent tactile access to the objects. During the tactile-only phases, a red-tinted light bulb $(40 \mathrm{~W})$ mounted to the tripod above the apparatus was used to light the testing area, and the plastic barrier was removed. All rats were habituated to the Y-shaped apparatus over two consecutive days. In counterbalanced order on both days, each rat spent $5 \mathrm{~min}$ in the Y-shaped apparatus in red light and $5 \mathrm{~min}$ in the apparatus in white light with the transparent plastic barriers in place (Fig. 1). During habituation, no objects were placed in the apparatus. Each rat was individually brought to the experiment room in a transport cage, placed in the start box of the Y-shaped apparatus, and released into the main exploration area for $5 \mathrm{~min}$ for each habituation session.

Behavioral testing began at least $24 \mathrm{~h}$ after the second habituation session. Each trial consisted of two phases, sample and choice, separated by a retention delay ( $1 \mathrm{~h}$ in length for all experiments except for the middle testing phase of the crossed unilateral lesion experiment, for which the retention delay was $60 \mathrm{~s}$ ). For the exclusively behavioral experiments, each rat was run for at least one trial. For all lesion experiments 
a Tactile SOR

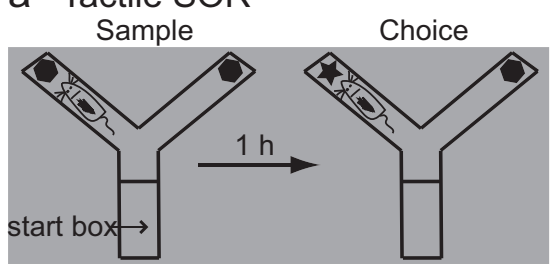

b Visual SOR

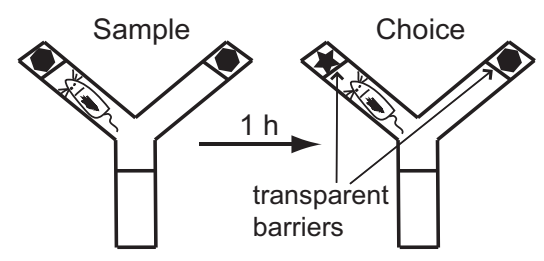

C Crossmodal object recognition

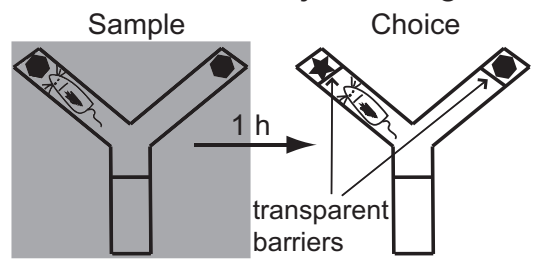

Figure 1. Schematic representation of the three SOR tasks used in this study. $\boldsymbol{a}$, In the tactile SOR task, the sample and choice phases are run in red light (indicated by shading) to prevent rats from using the visual properties of the objects. Thus, animals must recognize the objects on the basis of tactile qualities. $\boldsymbol{b}$, In the visual SOR task, the sample and choice phases are run in white light, and transparent plastic barriers inserted in the Y-shaped apparatus prevent physical contact with the objects. Thus, rats must encode and recognize the visual characteristics of the objects. c, In the CMOR task, rats experience a tactile-only sample phase in red light (indicated by shading) followed by a visual-only choice phase in white light with the transparent barriers inserted. Thus, animals must be able to recognize the visual qualities of the object in the choice phase on the basis of a tactile representation acquired in the sample phase.

except the unilateral lesion experiments, each rat was run for two trials on each of the three tasks; in the unilateral lesion experiments, rats were run for two trials on the crossmodal task only. A different object pair was used for each trial for a given rat, and the order of exposure to object pairs as well as the designated sample and novel objects for each pair were counterbalanced. Furthermore, the object pairs were counterbalanced across tasks (tactile, visual, crossmodal) within each experiment, such that, for example, one rat might have object pairs A and D for its two tactile-only SOR trials whereas another rat could have object pairs B and E. This was done, in part, to reduce the possible influence of the specific sensory characteristics of particular objects on specific tasks. The same object sets were used for all lesion experiments. Data were collected by scoring exploratory bouts using a personal computer.

In the sample phase, two identical objects were placed in the Y-shaped apparatus, one at the end of each exploration arm. Each rat was individually brought into the experiment room in a transport box and placed in the start box with the guillotine door lowered. The door was then raised to allow the rat into the exploration area of the maze. The time spent exploring the two objects was scored by an experimenter viewing the rat on a video screen. Exploration of an object was defined as directing the nose to the object, or the area of the transparent barrier within the outline of the object in the visual-only condition, at a distance of $<2 \mathrm{~cm}$ and/or touching it with the nose or whiskers. The sample phase ended when the rat had explored the identical objects for a total of $25 \mathrm{~s}$ or after $3 \mathrm{~min}$ had passed, whichever came first. At the end of the sample phase, the rat was removed from the $\mathrm{Y}$-shaped apparatus and transported back to its home cage.

In the choice phase, the Y-shaped apparatus contained a triplicate version of the sample ("familiar") object in one arm and a new object in the other. The exploration arms in which the choice objects were placed were counterbalanced between rats and across trials. The time spent exploring the novel and familiar objects was recorded for the $1 \mathrm{~min}$ choice phase. We then calculated a discrimination ratio, the difference in time spent exploring the novel and familiar objects divided by the total time spent exploring the objects, for each object recognition trial. This measure takes into account individual differences in the total amount of exploration time. Normal rats tend to explore the novel object more than the familiar sample object in this spontaneous object recognition paradigm.

Red versus white light. To test that the red light condition used for the tactile-only aspects of the unimodal and crossmodal SOR tasks was sufficient to prevent access to the visual properties of objects, we ran two groups of rats in an experiment using tactually identical but visually distinct stimuli (distinctively painted patterned vases, identical in size and shape). Six rats were tested for SOR performance, as described above, exclusively in the red light condition, in which the testing room was darkened in the sample and choice phases except for a single $40 \mathrm{~W}$ red light lamp suspended above the Y-shaped apparatus. Another six rats were tested in the exact same manner except with normal white overhead lighting during the sample and choice phases. Supplemental Figure 1 (available at www.jneurosci.org as supplemental material) shows the results of this experiment.

Tactile-only (unimodal) SOR. Rats were run with tactually and visually distinct junk objects according to the SOR procedure described above, but with red overhead lighting throughout all testing phases (Fig. 1a). An experiment in which six rats were tested in this manner confirmed their ability to perform tactile-only SOR (supplemental Fig. 2, available at www.jneurosci.org as supplemental material).

Visual-only (unimodal) SOR. Rats were run with the same junk objects used for the tactile SOR task. The standard SOR procedure, as described above, was used, except that access was limited to the visual properties of the objects by placing transparent plastic barriers between the rats and the objects to prevent animals from directly contacting the objects (Fig. $1 b)$. An experiment in which six rats were tested in this manner confirmed their ability to perform visual-only SOR (supplemental Fig. 2, available at www.jneurosci.org as supplemental material).

Preventing rats from touching the objects resulted in significant reductions in overall object exploration times in the visual-only condition, particularly in the choice phase (data not shown; see supplemental Table 3 , available at www.jneurosci.org as supplemental material, for data from lesion experiments). This means that relatively small differences in novel and familiar exploration times could yield robust object recognition (discrimination ratio) scores. Thus, to preclude the possibility that scorer bias or error might have substantially influenced the data, in addition to the procedural details described above, the original experimenter (J.M.R.) and an additional researcher scored all of the data off-line from this and all subsequent experiments. Both scorers were blind to the lesion group assignment for each subject, as well as the identity of the novel and familiar object for each individual trial. The interrater reliability for choice phase scoring was highly significant (Pearson $r=0.4-0.85$ ) for all task types in all experiments. Thus, it is unlikely that any observer bias substantially influenced the data reported in the following sections.

CMOR. Rats were run with the same junk objects used for the tactile and visual SOR tasks. A tactile-to-visual CMOR task was devised by combing the unimodal tasks described above. The sample phase was run in red light without the barriers, and the choice phase was run in white light with the barriers in place to prevent physical contact with the objects (Fig. 1c). An experiment in which five rats were tested in this manner confirmed their ability to perform crossmodal tactile-to-visual SOR (supplemental Fig. 2, available at www.jneurosci.org as supplemental material).

Data analysis. For the red versus white light, tactile-only, visual-only, and CMOR behavioral experiments, two-tailed one-sample $t$ tests were used to analyze the discrimination ratio (object recognition) data. The mean discrimination ratio in each condition was compared with zero, the value when rats show no preference between the novel and familiar object in the choice phase. Group means of four measures taken from object recognition testing were analyzed for the bilateral PRh, PPC, and crossed unilateral lesion experiments. Two measures were analyzed from each 
phase of behavioral testing: the total object exploration in the sample phase and the duration of the sample phase and the total object exploration and discrimination ratio from the choice phase. The total exploration and duration control measures (see supplemental Tables 1-3, available at www.jneurosci.org as supplemental material) were assessed to ensure the absence of any lesion-induced differences in general exploratory behavior in the sample or choice phases. Means from these four measures were submitted to two-way (task $\times$ lesion group) mixed-factorial ANOVAs, and planned comparison post hoc independent-samples $t$ tests were used to assess group differences within tasks. Univariate (lesion group) ANOVAs were used to analyze the same four behavioral measures for the unilateral lesion control experiments. All statistical analyses were conducted with a significance level of $\alpha=0.05$ using SPSS 16.0 for Windows.

\section{Results}

\section{Histology}

For all lesion studies described below, the excitotoxin-induced brain damage was centered on the targeted structures, with only minimal unintended damage to nearby regions seen in a few cases. Figure 2 illustrates the extent of PRh damage observed in the bilateral PRh lesion experiment. Cell loss was observed throughout the rostral-caudal extent of PRh in all rats with intended PRh damage. Unilateral $\mathrm{PRh}$ lesions in the disconnection experiment and the unilateral lesion control experiments were highly similar to those shown in Figure 2. The estimated average percentage of lesioned PRh area in the bilateral PRh lesion experiment was 87\% (range: 69-98\%). In the disconnection experiment, average unilateral PRh damage was estimated at $96 \%$ (range: $87-100 \%$ ) of control values. In the two unilateral control experiments, average unilateral PRh damage was estimated at $97 \%$ (separate unilateral lesions; range: $91-100 \%$ ) and $92 \%$ (ipsilateral unilateral lesions; range: 81-100\%). In cases in which targeted PRh tissue was intact, this tended to involve sparing of the most rostral PRh regions. In addition to the intended damage to PRh, two rats in the bilateral $\mathrm{PRh}$ experiment and three in the disconnection experiment also sustained unilateral damage to the lateral entorhinal cortex (all in the left hemisphere). Two rats in the bilateral PRh experiment also had very minor unilateral damage to area CA1 in the ventral hippocampus.

Figure 3 shows the extent of PPC damage seen in the bilateral PPC lesion experiment. The damage illustrated in Figure 3 at 5.3 $\mathrm{mm}$ caudal to bregma was observed in only one rat in the entire study. Cell loss was observed throughout the rostral-caudal extent of PPC in all rats in PPC lesion groups. Unilateral PPC lesions in the disconnection experiment and the unilateral lesion control experiments were very similar to those shown in Figure 3. The estimated average PPC lesion size in the bilateral PPC lesion experiment was $84 \%$ (range: $56-100 \%$ ). In the disconnection experiment, average unilateral PPC damage was estimated at 91\% (range: $70-100 \%$ ). In the two unilateral control experiments, average unilateral PPC damage was estimated at 99\% (separate unilateral lesions; range: $92-100 \%$ ) and 96\% (ipsilat-

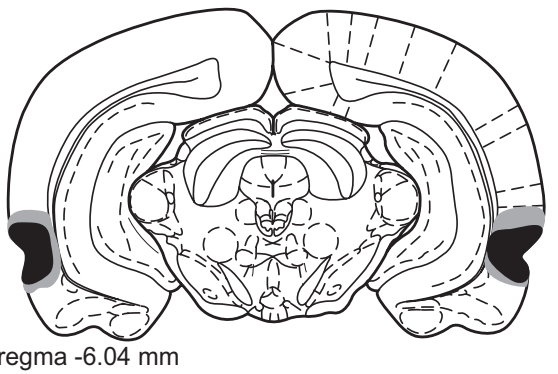

Bregma $-6.04 \mathrm{~mm}$

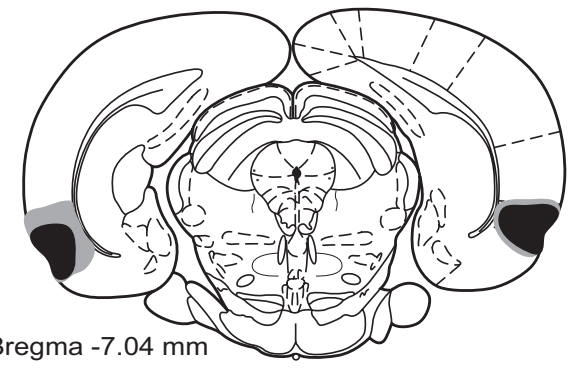

Bregma $-7.04 \mathrm{~mm}$

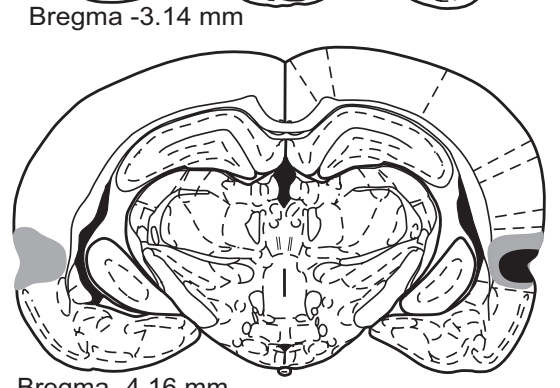

Figure 2. Infusions of the excitotoxin NMDA caused extensive cellular damage throughout PRh. Coronal sections illustrating the extent of the largest (gray) and smallest (black) lesions of PRh, from 3.14 to $7.04 \mathrm{~mm}$ posterior to bregma (Paxinos and Watson, 1998), in the bilateral PRh lesion experiment.

eral unilateral lesions; range: $88-100 \%)$. In addition to intended PPC damage, two rats in each of the bilateral PPC, unilateral PPC, and disconnection lesion groups also had minor unilateral damage to the CA1 region of the dorsal hippocampus associated with the PPC lesion procedure. Three rats in the ipsilateral unilateral lesion group had similar minor dorsal hippocampus damage.

\section{Behavioral assessment of crossmodal and unimodal spontaneous object recognition in rats}

To assess CMOR in rats, we developed three different variants of the SOR task (Ennaceur and Delacour, 1988), which exploits the natural tendency of rats to explore novel versus familiar objects. In the standard version of SOR, rats are presented with a pair of identical junk objects to explore in a sample phase; then, after a retention delay, an identical triplicate of the sample object is presented along with a novel object in the choice phase. Normal rats preferentially explore the novel object, thereby demonstrating their recognition of the sample object. This task is often considered to be mediated primarily by visual information processing; however, rats regularly touch the objects with their paws and whiskers while exploring. We therefore exploited this fact to develop separate unimodal (tactile and visual) and crossmodal (tactile-to-visual) versions of the task. The retention delay between sample and choice phases was $1 \mathrm{~h}$ for all tasks, and the same three-dimensional junk objects were used for all versions. In the tactile SOR task, rats were tested in red light for all phases (Fig. 1a). An initial behavioral experiment indicated that this condition was sufficient to prevent access to the visual properties of the objects (supplemental Fig. 1, available at www.jneurosci.org as 


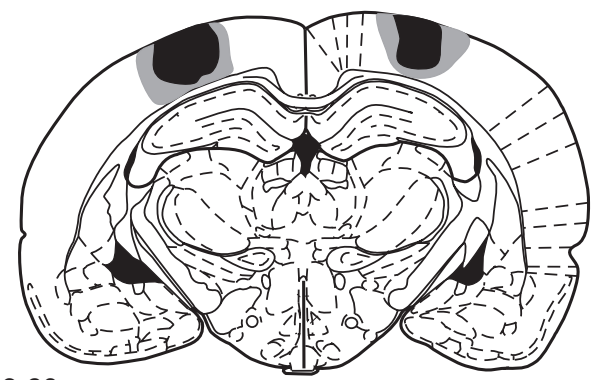

Bregma $-3.60 \mathrm{~mm}$

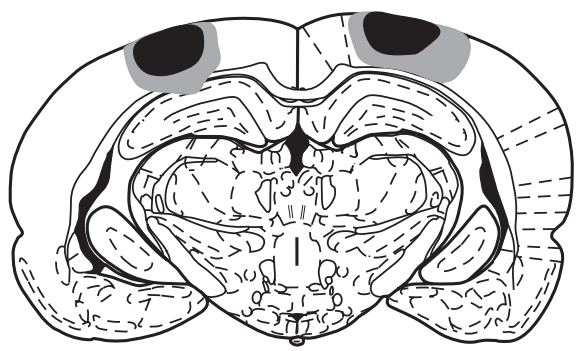

Bregma $-4.16 \mathrm{~mm}$

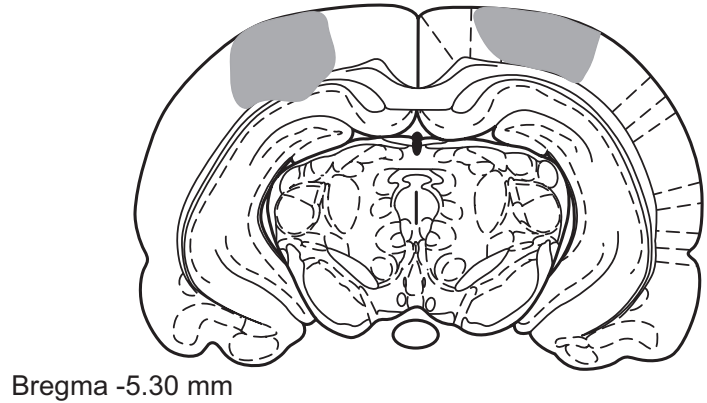

Figure 3. Infusions of the excitotoxin NMDA caused extensive cellular damage throughout PPC. Coronal sections illustrating the extent of the largest (gray) and smallest (black) lesions of PPC, from 3.6 to $5.3 \mathrm{~mm}$ posterior to bregma (Paxinos and Watson, 1998), in the bilateral PPC lesion experiment.

supplemental material). Rats tested in the tactile SOR task demonstrated a clear preference for the novel object, indicating good tactile recognition memory (one-sample $t$ test vs zero: $t_{(5)}=7.03$, $p=0.001$; supplemental Fig. 2, available at www.jneurosci.org as supplemental material). While contribution of olfactory cues left by the rat in the sample phase can be ruled out, as duplicates of the sample objects were presented in the choice phase, it is possible that the specific olfactory characteristics of the objects could be used by the rats to guide recognition performance in the choice phase of the tactile SOR task. While this must be borne in mind, the potential contribution of olfactory cues to recognition in this task is likely to be minor, and even less so in the crossmodal task (below), in which the animals are prevented from contacting the objects in the choice phase.

For the visual SOR task, rats were tested in white light for all phases as in the standard SOR procedure, but transparent barriers prevented the animals from touching the objects (Fig. 1b). Rats tested in this task showed strong visual object recognition memory (one-sample $t$ test vs zero: $t_{(5)}=3.29, p=0.022$; supplemental Fig. 2, available at www.jneurosci.org as supplemental material).

For the CMOR task, rats were run in red light (tactile-only) for the sample phase but in white light with the transparent barriers (visual-only) for the choice phase (Fig. 1c). Rats displayed robust crossmodal object recognition abilities (one-sample $t$ test vs zero:

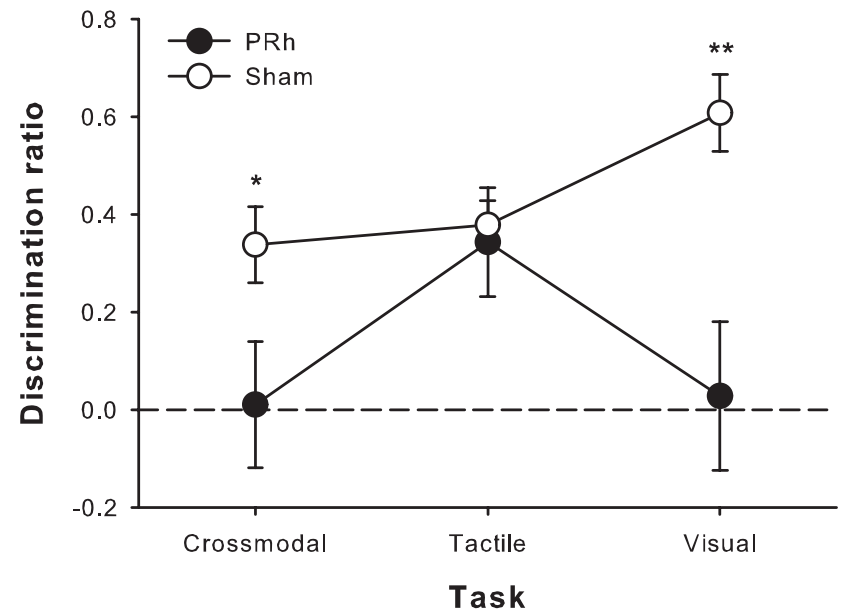

Figure 4. Bilateral excitotoxic PRh lesions significantly disrupted visual and crossmodal object recognition with a $1 \mathrm{~h}$ retention delay between the sample and choice phases but did not affect tactile recognition. Data are presented as average discrimination ratio ( \pm SEM). Dashed line (0) indicates equal preference for sample and novel objects. ${ }^{*} p<0.05$; ${ }^{* *} p<0.01$.

$t_{(4)}=7.23, p=0.002$; supplemental Fig. 2, available at www. jneurosci.org as supplemental material). This is especially impressive considering the fact that animals never experience both the tactile and visual properties of the objects simultaneously. Thus, unlike much of the nonhuman primate literature, there is no opportunity for the animals to form a crossmodal association from prior experience (Murray et al., 1998). This finding indicates that rats are capable of performing a spontaneous or automatic crossmodal comparison to facilitate the object recognition process.

\section{Bilateral perirhinal cortex lesions disrupt visual and crossmodal object recognition}

Having demonstrated the proficiency of rats in the unimodal and crossmodal object recognition tasks, we next assessed the neuroanatomical basis of these abilities. Previous findings from humans and monkeys suggest that PRh is importantly involved in crossmodal analysis of object features (Goulet and Murray, 2001; Taylor et al., 2006; Holdstock et al., 2009). Because of its established role in object information processing, we predicted that $\mathrm{PRh}$ lesions would disrupt the performance of rats in all three versions of SOR as assessed in the current study; however, no data currently exist to speak to the role of the rodent $\mathrm{PRh}$ in tactile or crossmodal object recognition.

Rats were randomly assigned to receive bilateral excitotoxic lesions of PRh (Fig. 2) or sham surgeries and, after 7-10 d of recovery, were tested on all three versions of SOR in counterbalanced fashion. Lesioned animals were significantly impaired on the visual and crossmodal tasks but performed the same as shams on the tactile task (mixed-factors ANOVA, task, $F_{(2,28)}=2.43$, $p=0.107$, group, $F_{(1,14)}=15.35, p=0.002$, task $\times$ group interaction, $F_{(2,28)}=4.71, p=0.017$; post hoc independent-samples $t$ tests, visual: $t_{(14)}=3.9, p=0.002$; CMOR: $t_{(14)}=2.46, p=0.028$; tactile: $t_{(14)}=0.34, p=0.74$ ) (Fig. 4). Although there were significant differences between tasks in terms of general object exploration (sample duration, total sample object exploration, and total choice object exploration), the lesion group and task $\times$ lesion group interaction terms were not significant for any of these control measures. This was also the case for all subsequent lesion experiments described (see supplemental Tables 1-3, 


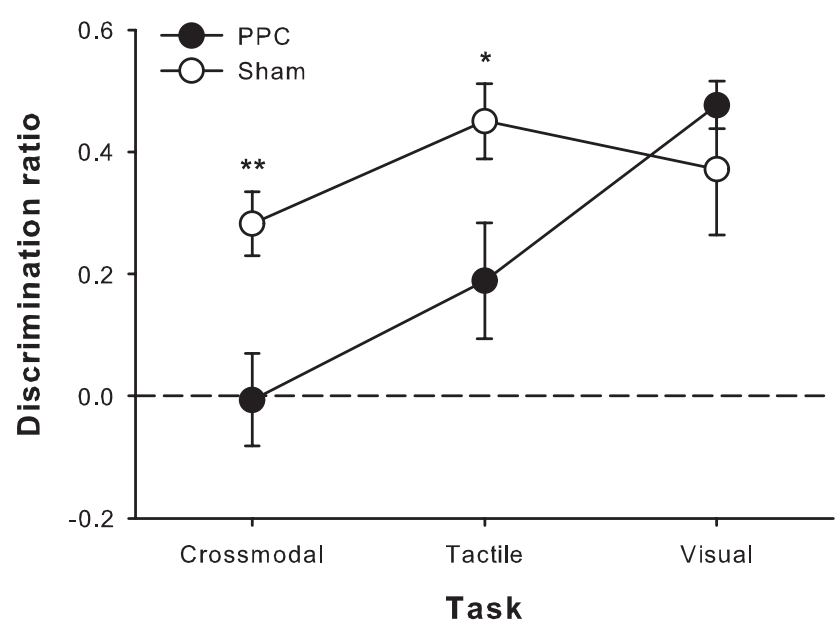

Figure 5. Bilateral excitotoxic PPC lesions significantly impaired tactile and crossmodal object recognition with a $1 \mathrm{~h}$ retention delay, leaving visual recognition intact. Data are presented as average discrimination ratio ( \pm SEM). Dashed line $(0)$ indicates equal preference for sample and novel objects. ${ }^{*} p<0.05 ;{ }^{* *} p<0.01$.

available at www.jneurosci.org as supplemental material, for control measure data and statistics).

\section{Bilateral posterior parietal cortex lesions disrupt tactile and crossmodal object recognition}

In light of previous results with monkeys (Goulet and Murray, 2001), we were somewhat surprised to find that PRh lesions did not disrupt tactile object recognition in rats. The pattern of impairment with $\mathrm{PRh}$ lesions does not allow a firm conclusion that $\mathrm{PRh}$ is the locus of a multimodal object representation. Indeed, this pattern suggests that PRh may be involved in the CMOR task by virtue of its role in visual stimulus representation. We thus sought to determine which brain region might contribute analogously to the tactile component of the CMOR task. The rat PPC is an area of association cortex that receives highly processed information from somatosensory, auditory, and visual areas (Reep et al., 1994) and may therefore be important for higher-order cognitive functions involving multiple sensory modalities. Accordingly, cells in somatosensory parietal cortex appear to represent visual-tactile crossmodal associations in monkeys (Zhou and Fuster, 2000).

A new group of rats therefore received either bilateral excitotoxic lesions of PPC (Fig. 3) or sham surgeries and were then tested on all three versions of SOR in counterbalanced fashion. Lesioned animals were significantly impaired on the tactile and crossmodal tasks but performed as well as shams on the visual task (mixed-factors ANOVA, task, $F_{(2,28)}=8.001, p=0.002$, group, $F_{(1,14)}=6.15, p=0.026$, task $\times$ group interaction, $F_{(2,28)}=4.58, p=$ 0.019 ; post hoc independent-samples $t$ tests, tactile: $t_{(14)}=2.57, p=$ 0.022; CMOR: $t_{(14)}=3.47, p=0.004$; visual: $t_{(14)}=0.87, p=0.401$ ) (Fig. 5). This pattern of results implies that PPC contributes important tactile information processing for successful object recognition when tactile information is critical and that this contribution is important for crossmodal (tactile-to-visual) object recognition as well.

Although Figure 5 appears to show some residual tactile SOR ability in PPC-lesioned rats, a one-sample $t$ test indicated that this performance was not significantly different from chance (discrimination ratio of zero; $\left.t_{(6)}=2.14, p=0.075\right)$. This trend toward intact tactile SOR in PPC-lesioned rats, compared with the complete abolition of CMOR performance in the same animals, raises the possibility that the CMOR task is more demand- ing on PPC processing. This potential tactile SOR sparing is not statistically significant, however, and will require further investigation for firm conclusions to be drawn.

\section{PRh and PPC functionally interact to mediate crossmodal object recognition}

The preceding results suggest that $\mathrm{PRh}$ and $\mathrm{PPC}$ are components of a distributed network involved in crossmodal object recognition. We therefore next asked whether a functional interaction could be demonstrated between PRh and PPC in the CMOR task. We hypothesized that rats with crossed unilateral lesions of PRh and PPC would be selectively impaired on the CMOR task. The crossed unilateral lesions should produce a disconnection within the putative functional circuit by disrupting visual information processing in one hemisphere (PRh lesion) and tactile information processing in the other (PPC lesion). A new group of rats thus received either sham surgery or crossed unilateral lesions of PRh and PPC and were subsequently tested on the three object recognition tasks. Lesioned animals were selectively impaired on the CMOR task, showing no signs of impairment on the tactile or visual unimodal tasks (planned comparisons: CMOR: $t_{(18)}=$ $2.11, p=0.0491$; visual: $t_{(18)}=0.69, p=0.496$; tactile: $t_{(18)}=$ $0.16, p=0.879$ ) (Fig. 6a).

To analyze further the nature of the selective CMOR deficit produced by crossed unilateral PRh and PPC lesions, we retested the same animals on the three tasks, but with a $60 \mathrm{~s}$ retention delay between the sample and choice phases. As the PRh and PPC have been implicated in perceptual and attentional processes, respectively (King and Corwin, 1993; Buckley and Gaffan, 2006; Bussey and Saksida, 2007; Bucci, 2009; Reep and Corwin, 2009), we wished to determine whether the CMOR deficit reported here could be considered a specifically mnemonic impairment rather than a disruption in visual and tactile perception or attention. Indeed, lesioned rats demonstrated intact object recognition on all three tasks when tested with the shorter retention delay (mixed-factors ANOVA, task, $F_{(2,36)}=2.18, p=0.128$, group, $F_{(1,18)}=0.002, p=0.968$, task $\times$ group, $\left.F_{(2,36)}=0.501, p=0.61\right)$ (Fig. 6b).

To ensure that unimpaired performance with the shorter retention delay was not attributable to recovery of function over time, we tested the same animals once more with the original $1 \mathrm{~h}$ retention delay, and the selective CMOR impairment was once again evident (mixed-factors ANOVA, task, $F_{(2,36)}=4.96, p=$ 0.013 , group, $F_{(2,36)}=3.14, p=0.093$, task $\times$ group interaction, $F_{(2,36)}=3.57, p=0.038$; post hoc independent-samples $t$ test, CMOR: $t_{(18)}=2.9, p=0.01$; visual: $t_{(18)}=0.398, p=0.695$; tactile: $t_{(18)}=0.65, p=0.527$ ) (Fig. $6 c$ ). The delay-dependent nature of the CMOR impairment strongly suggests that PRh and PPC cooperate in guiding performance in this task and that their contributions go beyond merely perceiving visual and tactile information. It appears that PRh and PPC are components of a functional circuit required for the mnemonic processing of crossmodal information.

Although unlikely, it is possible that unilateral damage to either PRh or PPC could cause a selective CMOR impairment. Thus, in a separate control experiment, rats with unilateral PRh or unilateral PPC lesions were tested on the CMOR task with a $1 \mathrm{~h}$ retention delay. Lesioned animals performed the same as shamoperated rats (univariate ANOVA, $F_{(2,17)}=0.05, p=0.951$; mean discrimination ratio $\pm \mathrm{SEM}$ : sham $=0.30 \pm 0.07 ; \mathrm{PRh}=$ $0.30 \pm 0.07 ; \mathrm{PPC}=0.31 \pm 0.08$ ), indicating that separate unilateral damage to either region is insufficient to disrupt crossmodal object recognition memory. Finally, an additional control exper- 


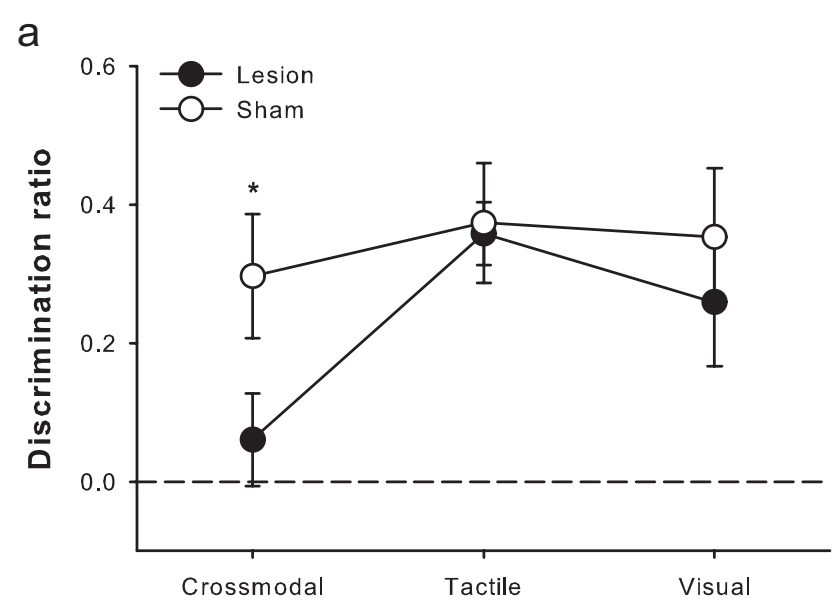

Task
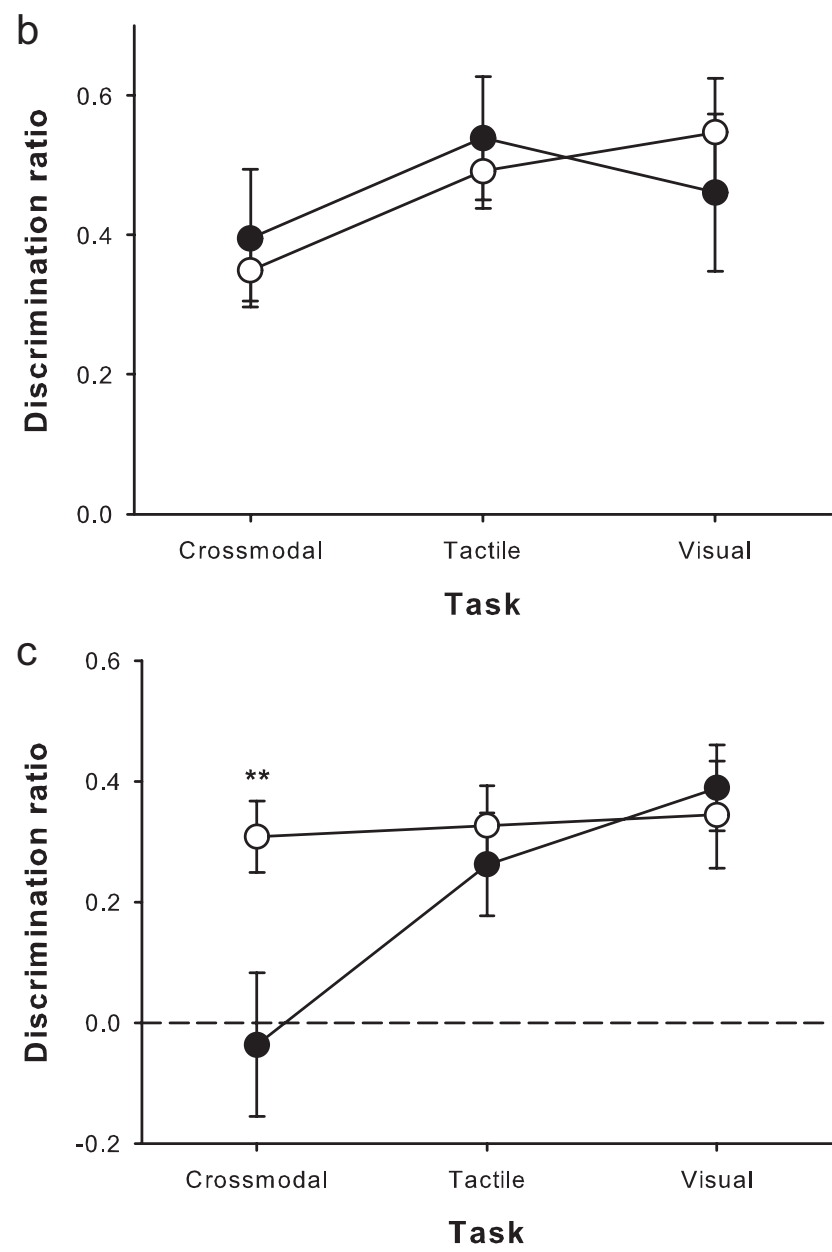

Figure 6. Crossmodal (tactile-to-visual) object recognition memory requires functional interaction between PRh and PPC. $\boldsymbol{a}$, Rats with crossed unilateral lesions of PRh and PPC displayed a selective deficit in crossmodal object recognition ( $1 \mathrm{~h}$ retention delay). $\boldsymbol{b}$, The impairment disappeared when the retention delay between sample and choice phases was reduced to $60 \mathrm{~s}$. c, The same animals were again impaired when retested on the CMOR task with a $1 \mathrm{~h}$ retention delay. Data are presented as average discrimination ratio ( \pm SEM). Dashed line $(0)$ indicates equal preference for sample and novel objects. ${ }^{*} p<0.05 ;{ }^{* *} p<0.01$.

iment assessed the possibility that combined ipsilateral unilateral PRh and PPC lesions could disrupt CMOR performance. Such a result would argue against functional interaction between these structures in the performance of the CMOR task. Lesioned ani- mals, however, were not impaired relative to sham performance (univariate ANOVA, $F_{(1,16)}=0.7, p=0.415$; mean discrimination ratio \pm SEM: sham $=0.28 \pm 0.09$; PRh plus PPC ipsilateral lesion $=0.37 \pm 0.07$ ). Together, these results strongly support the suggestion that successful crossmodal object recognition in the CMOR task requires functional interaction between PRh and PPC.

\section{Discussion}

The present results demonstrate that rats are capable of using exclusively visual or tactile information to recognize objects. Furthermore, our data show for the first time that rats are capable of crossmodal object recognition in the SOR paradigm. This is a potentially valuable animal model for use in the study of the neural underpinnings of crossmodal cognition and object feature binding, which are complex and integral cognitive abilities that remain poorly understood. The significance of this paradigm is further enhanced by the specific nature of the crossmodal task performed by rats in the present study. In the CMOR task, rats are not trained on any rule and never experience the visual and tactile properties of objects simultaneously; they are therefore never presented with the opportunity to form an explicit crossmodal association that could facilitate recognition memory when asked to recognize a visual stimulus on the basis of a stored tactile representation. It therefore seems that rats are capable of performing an "inferential" or automatic crossmodal comparison in the choice phase to demonstrate their recognition of the familiar object. This is a strikingly advanced cognitive ability for rodents, but one with obvious adaptive value.

The present study also provides important information about the neuroanatomical basis of this crossmodal object recognition ability. Our findings suggest that spontaneous tactile-to-visual CMOR task performance is guided by an object representation that is distributed between the perirhinal and posterior parietal cortices. Our results imply that $\mathrm{PRh}$ is particularly important for visual aspects of object processing in the SOR task, whereas PPC plays a vital role in analyzing tactile object features. The selective CMOR impairment produced by crossed unilateral lesions of these two cortical regions indicates that functional interaction between PRh and PPC is necessary for this type of crossmodal object memory. Moreover, the involvement of PRh and PPC in this task goes beyond simple sensory contributions, as the CMOR deficit produced by crossed unilateral lesions was only apparent with a relatively long retention delay. Thus, with at least a $1 \mathrm{~h}$ retention delay, PRh and PPC appear to cooperate to facilitate memory retention and retrieval in the service of crossmodal object recognition. The finding that rats with crossed unilateral lesions are not impaired at CMOR with a short retention delay is consistent with previous studies showing delay-dependent effects of PRh lesions in standard object recognition tasks (Winters et al., 2008), and recent suggestions that the hierarchical organization of the ventral visual stream involves compensatory mechanisms when perceptual or mnemonic abilities are not maximally taxed (Bussey and Saksida, 2007). The present results also suggest that similar principles may apply to PPC and its apparent role in processing tactile object information.

It remains to be seen whether the functional interaction demonstrated here is accomplished via direct communication between PRh and PPC or whether the information in these regions is fed into a common polymodal area, which could perform the necessary comparison between tactile and visual object properties or store an amodal object representation (Ettlinger and Wilson, 1990; Amedi et al., 2005; Lacey et al., 2007; Stein and 
Stanford, 2008). Studies from humans and monkeys suggest that prefrontal cortex may be a likely candidate to perform such functions (Banati et al., 2000; Fuster et al., 2000). It has also been suggested that $\mathrm{PRh}$, because of its established role in object identification, may be the repository of long-term crossmodal object representations (Murray et al., 1998; Goulet and Murray, 2001; Taylor et al., 2006). Data supporting these suggestions, however, come mainly from primate studies using crossmodal object recognition tasks in which the animals have extensive bimodal experience with the test objects. The present results are consistent with these past studies in implicating PRh in crossmodal object processing, although the specific role of PRh in the CMOR task may differ from other crossmodal tests used for other species. A very important related question concerns whether the spontaneous CMOR task described here, which does not involve simultaneous visual and tactile exposure, has different neural substrates than a task that encourages the formation of explicit crossmodal associations (Murray et al., 1998). Comparisons between early primate studies and more recent analyses in monkeys using different forms of crossmodal recognition tasks suggest that this may be the case (Ettlinger and Wilson, 1990; Murray et al., 1998). Moreover, the most parsimonious interpretation of the present data suggests that PRh contributes to performance in the rat CMOR task by virtue of its role in visual stimulus processing, not because it supports a multimodal object representation. It is possible that explicit crossmodal associations, such as those that could be expected to result from repeated simultaneous exposure to multimodal properties of an object, may be stored in a specialized brain region such as $\mathrm{PRh}$ or prefrontal cortex, whereas the more automatic type of crossmodal recognition demonstrated in the present study depends on a simpler mechanism involving comparison of information between (relatively) modalityspecific brain areas. Such a finding would help to explain the equivocal nature of the primate lesion literature on the neural bases of crossmodal object recognition. A second possibility regarding the apparent differential involvement of $\mathrm{PRh}$ in the rat CMOR task compared with monkey and human tests (Goulet and Murray, 2001; Holdstock et al., 2009) is that the rodent and primate brains process crossmodal object information in different ways.

We are currently performing experiments to address these important questions, and the paradigm introduced here will be highly valuable in the ongoing study of crossmodal recognition abilities in rodents. The results of the present study clearly show that a cortical network including PRh and PPC guides the spontaneous crossmodal object recognition ability of rats in a tactileto-visual CMOR task. These findings indicate that objects can be represented in a distributed manner within the cortex to facilitate performance of cognitive tasks requiring crossmodal information processing.

\section{References}

Amedi A, von Kriegstein K, van Atteveldt NM, Beauchamp MS, Naumer MJ (2005) Functional imaging of human crossmodal identification and object recognition. Exp Brain Res 166:559-571.

Banati RB, Goerres GW, Tjoa C, Aggleton JP, Grasby P (2000) The functional anatomy of visual-tactile integration in man: a study using positron emission tomography. Neuropsychologia 38:115-124.

Botly LC, De Rosa E (2007) Cholinergic influences on feature binding. Behav Neurosci 121:264-276.

Botly LC, De Rosa E (2009a) The nucleus basalis magnocellularis contributes to feature binding in the rat. Physiol Behav 97:313-320.

Botly LC, De Rosa E (2009b) Cholinergic deafferentation of the neocortex using 192 IgG-saporin impairs feature binding in rats. J Neurosci 29:4120-4130
Bucci DJ (2009) Posterior parietal cortex: an interface between attention and learning? Neurobiol Learn Mem 91:114-120.

Buckley MJ, Gaffan D (2006) Perirhinal cortical contributions to object perception. Trends Cogn Sci 10:100-107.

Burwell RD (2001) Borders and cytoarchitecture of the perirhinal and postrhinal cortices in the rat. J Comp Neurol 437:17-41.

Burwell RD, Amaral DG (1998) Perirhinal and postrhinal cortices of the rat: interconnectivity and connections with the entorhinal cortex. J Comp Neurol 391:293-321.

Burwell RD, Witter MP, Amaral DG (1995) Perirhinal and postrhinal cortices of the rat: a review of the neuroanatomical literature and comparison with findings from the monkey brain. Hippocampus 5:390-408.

Bussey TJ, Saksida LM (2007) Memory, perception, and the ventral visualperirhinal-hippocampal stream: thinking outside of the boxes. Hippocampus 17:898-908.

Chandler HC, King V, Corwin JV, Reep RL (1992) Thalamocortical connections of rat posterior parietal cortex. Neurosci Lett 143:237-242.

Cowey A, Weiskrantz L (1975) Demonstration of cross-modal matching in rhesus monkeys, Macaca mulatta. Neuropsychologia 13:117-120.

Davenport RK, Rogers CM (1970) Intermodal equivalence of stimuli in apes. Science 168:279-280.

Elliott RC (1977) Cross-modal recognition in three primates. Neuropsychologia 15:183-186.

Ennaceur A, Delacour J (1988) A new one-trial test for neurobiological studies of memory in rats. 1: Behavioral data. Behav Brain Res 31:47-59.

Ettlinger G, Wilson WA (1990) Cross-modal performance: behavioural processes, phylogenetic considerations and neural mechanisms. Behav Brain Res 40:169-192.

Forwood SE, Winters BD, Bussey TJ (2005) Hippocampal lesions that abolish spatial maze performance spare object recognition memory at delays of up to 48 hours. Hippocampus 15:347-355.

Fox MT, Barense MD, Baxter MG (2003) Perceptual attentional set-shifting is impaired in rats with neurotoxic lesions of posterior parietal cortex. J Neurosci 23:676-681.

Fuster JM, Bodner M, Kroger JK (2000) Cross-modal and cross-temporal association in neurons of frontal cortex. Nature 405:347-351.

Goulet S, Murray EA (2001) Neural substrates of crossmodal association memory in monkeys: the amygdala versus the anterior rhinal cortex. Behav Neurosci 115:271-284.

Holdstock JS, Hocking J, Notley P, Devlin JT, Price CJ (2009) Integrating visual and tactile information in the perirhinal cortex. Cereb Cortex 19:2993-3000.

Jarvis MJ, Ettlinger G (1977) Cross-modal recognition in chimpanzees and monkeys. Neuropsychologia 15:499-506.

Jeffery KJ (2007) Do discrimination tasks discourage multi-dimensional stimulus processing? Evidence from a cross-modal object discrimination in rats. Behav Brain Res 183:213-221.

Kesner RP (2009) The posterior parietal cortex and long-term memory representation of spatial information. Neurobiol Learn Mem 91:197-206.

King VR, Corwin JV (1993) Comparisons of hemi-inattention produced by unilateral lesions of the posterior parietal cortex or medial agranular prefrontal cortex in rats: neglect, extinction, and the role of stimulus distance. Behav Brain Res 54:117-131.

Lacey S, Campbell C, Sathian K (2007) Vision and touch: multiple or multisensory representations of objects? Perception 36:1513-1521.

Lipton PA, Alvarez P, Eichenbaum H (1999) Crossmodal associative memory representations in rodent orbitofrontal cortex. Neuron 22:349-359.

Murray EA, Mishkin M (1985) Amygdalectomy impairs crossmodal association in monkeys. Science 228:604-606.

Murray EA, Malkova L, Goulet S (1998) Cross-modal associations, intramodal associations, and object identification in macaque monkeys. In: Comparative neuropsychology (Milner AD, ed), pp 51-69. Oxford: Oxford UP.

Murray EA, Bussey TJ, Saksida LM (2007) Visual perception and memory: a new view of medial temporal lobe function in primates and rodents. Annu Rev Neurosci 30:99-122.

Over R, Mackintosh NJ (1969) Cross-modal transfer of intensity discrimination by rats. Nature 224:918-919.

Paxinos G, Watson C (1998) The rat brain in stereotaxic coordinates, Ed 3. London: Academic.

Reep RL, Corwin JV (2009) Posterior parietal cortex as part of a neural network for directed attention in rats. Neurobiol Learn Mem 91:104-113.

Reep RL, Chandler HC, King V, Corwin JV (1994) Rat posterior parietal 
cortex: topography of corticocortical and thalamic connections. Exp Brain Res 100:67-84.

Sahgal A, Petrides M, Iversen SD (1975) Cross-modal matching in the monkey after discrete temporal lobe lesions. Nature 257:672-674.

Sakata S, Yamamori T, Sakurai Y (2004) Behavioral studies of auditoryvisual spatial recognition and integration in rats. Exp Brain Res 159:409-417.

Stein BE, Meredith MA (1993) The merging of the senses. Cambridge, MA: MIT.

Stein BE, Stanford TR (2008) Multisensory integration: current issues from the perspective of the single neuron. Nat Rev Neurosci 9:255-266.

Taylor KI, Moss HE, Stamatakis EA, Tyler LK (2006) Binding crossmodal object features in perirhinal cortex. Proc Natl Acad Sci U S A 103:8239-8244.
Tomie JA, Whishaw IQ (1990) New paradigms for tactile discrimination studies with the rat: methods for simple, conditional, and configural discriminations. Physiol Behav 48:225-231.

Winters BD, Forwood SE, Cowell RA, Saksida LM, Bussey TJ (2004) Double dissociation between the effects of peri-postrhinal cortex and hippocampal lesions on tests of object recognition and spatial memory: heterogeneity of function within the temporal lobe. J Neurosci 24:5901-5908.

Winters BD, Saksida LM, Bussey TJ (2008) Object recognition memory: neurobiological mechanisms of encoding, consolidation and retrieval. Neurosci Biobehav Rev 32:1055-1070.

Zhou YD, Fuster JM (2000) Visuo-tactile cross-modal associations in cortical somatosensory cells. Proc Natl Acad Sci U S A 97:9777-9782. 